\title{
JURISPRUDENCIA AMBIENTAL EN CANARIAS (PRIMER SEMESTRE 2019)
}

\author{
ADOLFO JIMÉNEZ JAÉN \\ Profesor titular de Derecho Administrativo \\ Universidad de Las Palmas de Gran Canaria
}


Sumario: 1. Aprobación de planeamiento urbanístico: omisión del informe de costas. 2. Silencio administrativo en licencia de apertura. 3. Ocupación del dominio público: necesidad de instalación en el mismo. 4.- Autorización ambiental integrada de central diésel.

\section{APROBACION DE PLANEAMIENTO URBANÍSTICO: OMISIÓN DEL INFORME DE COSTAS.}

La sentencia del Tribunal Superior de Canarias de 14 de mayo de 2018 tiene como objeto la desestimación del requerimiento efectuado por la Administración General del Estado del acuerdo adoptado por la Comisión de ordenación del territorio y medio ambiente de Canarias, con fecha 29/07/14, relativo a la aprobación definitiva del plan general de ordenación supletorio de Yaiza (BOC no 152, de 26 de noviembre de 2014).

Concretamente la pretensión se refiere a la aprobación del Plan General sin que hubiera transcurrido el plazo que establece la Ley de Costas para que la Administración General del Estado emita dicho informe.

Lo primero que hay que destacar es que la Comunidad Autónoma envió en diversas ocasiones el Plan para que la Administración General del Estado emitiera su informe: La primera de ellas tiene lugar con ocasión del avance; así, con fecha 30 de agosto de 2010, el Gobierno de Canarias remite a la Dirección General de Costas, a través de la Demarcación de Costas de Canarias, el Avance del Plan General Supletorio de Yaiza. Con fecha 23 de febrero de 2012, el Gobierno de Canarias remite a la Dirección General, a través de la Demarcación de Costas de Canarias, el Plan General de Ordenación supletorio del municipio de Yaiza, solicitando el informe previsto en el artículo 117.1 de la Ley 22/1988, de 28 de julio, de Costas. La documentación se presenta sin diligenciar y sin constar el estado de la tramitación.

Con ocasión de esta solicitud, con fecha 25 de mayo de 2012, la Dirección General de Sostenibilidad de la Costa y el Mar emite informe señalando una serie de deficiencias fundamentalmente en tres aspectos: la representación de las líneas de ribera del mar, deslinde de dominio público y servidumbre de protección de los deslindes vigentes en el municipio; en la justificación de lo establecido en la Disposición Transitoria tercera 3.2 de la Ley de Costas para 
las fachadas marítimas de Playa Blanca y Playa Quemada, y en la no justificación del cumplimiento del artículo 30 de la Ley de Costas para los sectores de suelo urbanizable sectorizado, solicitando nuevamente que el expediente corregido. completo diligenciado previamente a su aprobación definitiva se remita de nuevo para emisión del informe de acuerdo con lo dispuesto en los artículos 112.a 117.2 de la Ley de Costas.

Con fechas Con fecha 15 de octubre de 2012, y 25 de febrero de 2014 el Gobierno realiza nuevas solicitudes de informe, trámite que se reitera el 15 de abril de 2014, Finalmente el 6 y 10 de junio de 2014 se envía información complementaria.

Pues bien la razón de la anulación del Plan general es el incumplimiento del plazo de dos meses que establece la Ley de Costas para la emisión del informe ya que entre se realiza los días 6 y 10 de junio de 2014 ( folios 27297 y siguientes del EA) y que la aprobación definitiva tiene lugar por la Comisión de Ordenación del Territorio y Medio Ambiente de Canarias con fecha 29 de julio de 2014 (folio 30.701 y siguientes del expediente), por lo que no había transcurrido el plazo de dos meses a que se refiere el repetido art 117.2 de la Ley de Costas. Es evidente -dice el Tribunal-que "el día inicial del cómputo del plazo de dos meses debe referirse a tal fecha teniendo en cuenta que en ella se dice expresamente que se "remite información respecto a la solicitud del preceptivo informe previsto en el artículo 117.2 de la Ley 22/1988, de 28 de julio, de Costas, con carácter previo a su aprobación definitiva del Plan General de Ordenación supletorio del municipio de Yaiza, en Lanzarote, en tramitación desde esta Consejería, se adjuntan certificaciones expedidas por el Ayuntamiento de Yaiza respecto a la aprobación y servicios de los siguientes Planes Parciales del Municipio: x-Plan Parcial Castillo del Aguila. -Plan Parcial Montaña Roja-Plan Parcial Puerto Calero-Plan Parcial San Marcial del Rubicón -Plan Parcial Las Coloradas-Plan Parcial Costa Papagayo".

Por todo ello, la Sentencia procede a anular el Plan General, si bien matiza el alcance de dicha declaración: "Aun cuando la jurisprudencia ha resaltado que la omisión de un informe es causa de nulidad de pleno derecho, debe considerarse también que tal omisión debe acarrear la anulación del procedimiento y su reposición al momento oportuno para que pueda suplirse tal 
falta emitiendo el referido informe. Ponderando ambas afirmaciones en este caso la nulidad que declaramos no afecta a la totalidad del Plan General impugnado, sino tan solo a la parte que incide sobre el dominio publico marítimo terrestre y sus zonas de servidumbre a fin de que se solicite y emita el informe previsto en el art 117.2 de la Ley de Costas".

\section{SILENCIO ADMINISTRATIVO EN LICENCIA DE APERTURA.}

La sentencia del Tribunal Superior de Canarias de 25 de junio de 2018 resuelve el recurso de apelación contra la sentencia del Juzgado de lo Contencioso Administrativo número 6 de fecha 17 de mayo de 2016, por la que se desestima la resolución presunta del Ayuntamiento de La Palmas en virtud de la cual se desestimó el recurso de reposición presentado contra la resolución 35136, de diecinueve de noviembre de dos mil trece, de la Directora General de Edificación y Actividades del Ayuntamiento de Las Palmas de Gran Canaria, contra la resolución expresa de siete de agosto de dos mi catorce, de la Directora General de Edificación y Actividades del Ayuntamiento de Las Palmas de Gran Canaria, y contra la resolución expresa 39184, de diez de noviembre de dos mil catorce, de la Directora General de Edificación y Actividades del Ayuntamiento de Las Palmas de Gran Canaria, que declaró conformes a derecho las resoluciones administrativas impugnadas, por lo que debo confirmarlas y las confirmó.

Como puede observarse el objeto del recurso es doble: por un lado, el Ayuntamiento había concedido al recurrente la licencia de instalación solicitada y, en la misma resolución de concesión de dicha licencia, le indicó los requisitos que debía cumplir para la obtención de la licencia de apertura y el plazo en el que debía cumplimentarlos.

En segundo lugar, el recurrente, habiendo sido correctamente notificado e informado de los pasos a seguir, no presentó, hasta quince años después, solicitud de obtención de licencia de apertura, que fue objeto de declaración de caducidad. 
El recurrente, además alega la obtención de la licencia de apertura por silencio administrativo, cuestión que el mismo juzgado de instancia rechaza, pues en ningún momento dicha licencia había sido solicitada, por lo que no podía haberse obtenido por tal silencio.

El Tribunal Superior empieza analizando, en primer término, la legalidad de la resolución $n^{0}$ 39184, de 10 de noviembre de 2.014, por la que se declara caducada la licencia de instalación concedida por Decreto del Alcalde de 1.998, y el archivo de la solicitud de licencia de instalación, apertura y funcionamiento solicitada en noviembre de 2.013 .

Respecto de este punto, la sentencia señala que "la licencia de instalación concedida por Decreto de 1.998, lo fue para ejecución de obras conforme a un proyecto técnico, y, por si sola, no autoriza a iniciar ninguna actividad sin la previa constatación del cumplimiento de los requisitos del proyecto y del ordenamiento jurídico, sin perjuicio de que el transcurso de quince años supone, en principio, la desconexión temporal absoluta entre licencia de instalación y solicitud de puesta en funcionamiento, y, por tanto, priori, es lógico entender que lo más probable sea la imposibilidad legal de concesión de esta última en base a un proyecto autorizado cuando la petición tiene lugar en un marco normativo y temporal radicalmente distinto".

Sin embargo, señala también que "Ahora bien, lo que no es posible desconocer es que la actividad funcionó sin autorización de puesta en funcionamiento, esto es, sin control del cumplimiento de los requisitos de licencia de funcionamiento, y que funcionó también sin autorización de apertura de la actividad, si bien a dicha situación anormal contribuyó decisivamente el propio Ayuntamiento que durante muchos años toleró el funcionamiento de la actividad sin visita alguna de comprobación de la adecuación de la instalación al proyecto, y sin actuación alguna de intervención en forma de control mínimo de una actividad que solo tenía licencia de instalación y cuyo titular no había solicitado la puesta en funcionamiento".

Pues bien, a partir de esa situación, la sentencia señala dos consecuencias de la misma: 
- Por un lado, "no era posible declarar la caducidad de la licencia de instalación cuya concesión no contenía indicación alguna de tal caducidad por no solicitarse la puesta en funcionamiento, más cuando dicha puesta en funcionamiento tuvo lugar aunque sin la correspondiente comprobación. Esto es, se llevó a cabo la actividad para la que se había solicitado y concedido licencia de instalación y dicho funcionamiento con la tolerancia del propio Ayuntamiento.

- De otra parte, "en cuanto a la licencia de apertura, que fue la solicitada por el titular de la actividad, no era posible el archivo sino que correspondía al Ayuntamiento, en relación a una actividad que funcionaba sin autorización de actividad (hecho indiscutido) dar una respuesta a la concreta y clara petición de licencia de actividad, y dicha respuesta debió darse, a la fecha de la solicitud, conforme a la normativa vigente, que no era otra que la Ley la Ley 7/2011, de 5 de abril, de Actividades Clasificadas y Espectáculos Públicos y otras Medidas Administrativas Complementarias, de forma que correspondía al Ayuntamiento identificar el régimen de intervención a que quedaba sujeta la concreta solicitud de licencia de apertura y dar una respuesta a esa concreta petición, y no dar por supuesto que lo solicitado era otra cosa (la puesta en funcionamiento de la instalación) por la sencilla razón de que nunca solicitó la parte tal puesta en funcionamiento en relación a un taller que ya estaba en funcionamiento".

Por todo ello, la sentencia del Tribunal Superior de Justicia de Canarias anula la resolución n³ 39184, de 10 de noviembre de 2014, por la que se declara caducada la licencia de instalación concedida por Decreto del Alcalde de 1998, y el archivo de la solicitud de licencia de instalación, apertura y funcionamiento. $Y$ ello por dos motivos: En primer término, por cuanto no cabe declarar caducada una licencia ya otorgada, al margen de las consecuencias que puedan derivar de la puesta en funcionamiento de la instalación sin comprobación, consecuencias que nunca aplicó la Administración pese al transcurso de los años. $Y$ en segundo lugar, por cuanto se debió dar una 
respuesta expresa a la concreta solicitud, que era de licencia de apertura y dicha respuesta pasaba por la identificación del régimen de intervención a la vista de la actividad para la que se solicitaba la autorización.

La segunda cuestión para analizar es la de si se obtuvo la licencia de apertura por silencio administrativo.

Respecto de este tema, la sentencia rechaza dicha pretensión y ello porque, dice la resolución que "nunca se pudo haber obtenido la licencia de actividad (de apertura) por silencio pues ello es una ficción legal que autoriza a entender estimada la petición y, en el caso, la misma parte formuló más de una solicitud de licencia de apertura, y, no solo eso, sino que llegó a presentar declarar responsable previa al inicio de la actividad al amparo de la ley, lo que significa que ella misma no hizo valer el silencio".

Por otra parte, la resolución recuerda que, en cuanto al régimen de actos presuntos en dicho cuerpo legal, el artículo 19, decía literalmente:

"Transcurrido el plazo al que se refiere el artículo anterior sin que por el alcalde se resolviese sobre el otorgamiento de la licencia, se entenderá producido el acto con los siguientes efectos: a) Si el informe de calificación hubiese sido favorable, o condicionado al cumplimiento de determinadas medidas correctoras, se entenderá otorgada la licencia y, en su caso, sujeta al cumplimiento de éstas. b) Si la calificación o el informe sobre el emplazamiento hubieran sido desfavorables a la concesión de la licencia, se entenderá denegada ésta.

Por su parte, el artículo 20 sobre inactividad de la Administración, añadía:

"1. Transcurridos dos meses desde la fecha de la solicitud sin que se hubiere remitido al Cabildo insular el expediente, podrá el interesado alegar esta circunstancia ante dicha corporación, que se subrogará en la tramitación municipal, instruyendo el correspondiente expediente y dictando la resolución procedente.

2. A efecto de lo dispuesto en el apartado anterior, los interesados deberán, o acompañar con su escrito copia de los documentos 
presentados en el Ayuntamiento, o, al menos, indicar los datos identificativos de los mismos para su reclamación por el Cabildo insular.

3. El cabildo insular comunicará la subrogación al Ayuntamiento, debiendo abstenerse éste a partir de la fecha de recepción de la comunicación de realizar cualquier actuación relativa al expediente de solicitud de la licencia de actividad, remitiendo copia del mismo en el estado de tramitación en que se encuentre y de la documentación e informes que considere oportunos al Cabildo insular. La no remisión por el Ayuntamiento del expediente y documentación que se acompañe en ningún caso supondrá la paralización del expediente incoado por el Cabildo insular.

4. Si en el plazo de tres meses, contados a partir de la comunicación de la subrogación por inactividad, el Cabildo insular no resolviese sobre el otorgamiento de la licencia, se estará a lo dispuesto en el artículo anterior y, en su caso, a lo establecido por la legislación sobre el procedimiento administrativo común."

Por tanto, resuelve el Tribunal, "el silencio positivo, en relación con actividades clasificadas, necesitadas de calificación, entre las que todas las partes sitúan la actividad de taller de mecánica, parte del contenido del previo informe de calificación y, en caso de inactividad municipal en la remisión del expediente, lo que prevé la ley es lo que prevé la ley es la subrogación del Cabildo pero no el silencio positivo".

A todo ello se añade el cambio de régimen jurídico que se produce como consecuencia de la incorporación de la Directiva de Servicios al ordenamiento jurídico español, y más concretamente al autonómico. En este sentido se señala que "De todas formas, aunque la parte hace expresa referencia a la aplicación de dicha ley, la posibilidad de producción del acto por silencio debe valorarse conforme a la legislación vigente cuando se solicita la licencia de apertura, y dicha solicitud tuvo lugar bajo la vigencia de la Ley 7/2011, de 5 de abril, de actividades clasificadas y espectáculos públicos y otras medidas administrativas complementarias, en cuanto se trataba de una solicitud de licencia de actividad de fecha 6 de noviembre de 2013 (registro núm 167283), 
esto es, en una fecha en la que ya estaba vigente la nueva normativa reguladora de la intervención administrativa en función del tipo de actividad".

En conclusión, la sentencia rechaza la obtención de la licencia de apertura por silencio administrativo, no sin antes señalar el inadecuado funcionamiento del Ayuntamiento, ya que "era obligación municipal la tramitación de la solicitud de autorización de apertura, y sin perjuicio de que no era posible la obtención de la licencia por silencio por cuanto, cuando se trata de solicitudes de licencia de instalación de actividad clasificada, el silencio positivo va siempre unido al contenido del previo informe de calificación (art 24), de forma que en defecto de informe, y conforme al apdo 3 "En los demás supuestos no previstos en el apartado anterior, el transcurso del plazo para resolver y notificar la resolución facultará al interesado para entender desestimada la solicitud y deducir, frente a la denegación presunta, los recursos que legalmente procedan, y sin que ello obste al deber de la Administración de dictar resolución expresa".

Y la resolución concluye: "Vemos, por tanto, que la ley establece el silencio negativo en los demás supuestos, entre ellos, el que aquí se examina en el que se solicitó la licencia de apertura y la Administración no es que haya desestimado la solicitud, sino que, con abierta vulneración del derecho de defensa del interesado, interpretó que su solicitud era otra, y archivo la petición".

A continuación, procede a establecer las consocias de todo lo expuesto:

- Por lado, "estimar el recurso de apelación a los solos efectos de declarar la nulidad de las resoluciones recurridas, esto es, a los efectos de declarar la nulidad de la caducidad de la licencia de instalación de concedida por Decreto del Alcalde de 1.998, y la nulidad del archivo de la solicitud de licencia de instalación, apertura y funcionamiento, formulada el 6 de noviembre de 2.013, y ello a los efectos de que el Ayuntamiento tramite y de respuesta a la solicitud, presentada el 6 de noviembre de 2.013 (registro $n^{\circ}$ 167283) de licencia de actividad (apertura) de un taller de automóviles, y que lo haga conforme al régimen jurídico previsto en la Ley 7/2011, para actividades clasificadas". 
- Por otro, desestimar "Ia pretensión de haber obtenido la licencia de apertura por silencio, que nunca se produjo, ni se podía producir conforme a la normativa vigente, ni tampoco se hizo valer por el interesado, de lo que es aval suficiente el propio recurso contencioso-administrativo contra el archivo de la solicitud de la licencia de apertura".

\section{OCUPACIÓN DEL DOMINIO PÚBLICO: NECESIDAD DE INSTALACIÒN EN EL MISMO.}

La sentencia de 23 de julio de 2018 analiza la desestimación del recurso de alzada interpuesto contra la resolución del Servicio Provincial de Costas de Tenerife de fecha 5 de julio de 2017, confirmando dicho acto administrativo por ser ajustado a derecho. Dicho acto administrativo no es otro que la resolución de 5 de julio de 2017 por la que de deniega la autorización solicitada con fecha 3 de noviembre de 2016 para la temporada del 1 de enero al 31 de diciembre de 2017 para la instalación de un puesto de elaboración y venta de maíz a la barbacoa, con una ocupación de $7 \mathrm{~m}^{2}$ en la Playa de El Camisón, en el término municipal de Arona. Dicha autorización se había concedido en los 2 años anteriores.

La resolución que desestimó el recurso de alzada menciona con más detalle los argumentos esgrimidos por el Ayuntamiento de Arona al emitir el informe desfavorable y considera que las razones que fundamentaron la denegación fueron dos, que las instalaciones por su naturaleza pueden ubicarse fuera del dominio público marítimo terrestre y el citado informe desfavorable.

La representación procesal de la parte actora postula la nulidad de dichos actos por las consideraciones siguientes:

$1^{\circ}$ Porque la instalación no puede ubicarse fuera del dominio público ya que colinda con un paseo y las edificaciones que lindan con dicho paseo, lo que se observa claramente en las fotografías y planos adjuntados al expediente administrativo.

$2^{\circ}$ Porque no es cierto que se hay instalado o se pretenda instalar un grupo electrógeno, existen paneles solares instalados, porque la 
actividad es muy demandada por los turistas europeos y los usuarios de la playa.

$3^{\circ}$ Porque las anteriores solicitudes de autorización, desde el 2014, fueron concedidas en la misma ubicación sin que se haya justificado el cambio de criterio en tan poco tiempo, por lo que la resolución deviene en arbitraria.

La resolución judicial basa el rechazo al recurso presentado en dos motivos:

$1^{\circ}$.- Por un lado, la sentencia señala como argumento lo dispuesto en el artículo 61.2.b) del Reglamento de Costas que establece que "Las actividades o instalaciones a que se refiere el apartado anterior son: b) Las de servicio público o al público que, por la configuración física del tramo de costa en que resulte necesario su emplazamiento, no puedan ubicarse en los terrenos colindantes con dicho dominio". En este sentido, se señala lo siguiente: "Ya hemos señalado antes que la posibilidad de autorizar en este caso la ocupación del DPMT deriva del apartado 2, b) del art.61 antes citado, no se trata del otro supuesto en que lo determinante es la función realizada que implica la necesidad de ocupar el DPMT, aquí se trata de determinar las características físicas del tramo de costa en el que se pretende ocupar el DPMT y la imposibilidad de ubicar la actividad fuera del DPMT. La parte señala que, con la documentación aportada y fotografías presentadas, resulta acreditado que no cabe realizar la ubicación en otro sitio en la zona en cuestión. La realidad es que, examinada la documentación y fotografías, ello no pude estimarse plenamente acreditado. Es verdad que la administración simplemente afirma que ello es posible y no presenta prueba alguna, pero lo cierto es que la prueba del hecho que determina la posibilidad de incluir la autorización en los supuestos legales a que se refiere el art. 61 corresponde a la parte que solicita la autorización. También se alega que no se ha justificado el cambio de criterio respecto a los años anteriores, lo cual, en cuanto a la ubicación de la actividad es cierto".

$2^{\circ}$.- En segundo término, el segundo motivo de la denegación de la autorización es el informe desfavorable del Ayuntamiento. Dicho informe menciona una superficie que no es real $\left(16,90 \mathrm{~m}^{2}\right)$, ni es la que se menciona en 
la solicitud presentada, son $7 \mathrm{~m}^{2}$. Además, señala la sentencia, "el hecho de que al Ayuntamiento pudiera interesarle que la actividad en cuestión se realice en fiestas locales en los diferentes núcleos a lo largo del calendario anual según los programas de fiestas locales es absolutamente irrelevante a efectos de la ocupación del DPMT, lo que le interese al Ayuntamiento en relación con sus fiestas locales no tiene nada que ver con lo que aquí ha de resolverse. Pese a lo anterior, también se menciona como base del informe desfavorable la previsión de instalación de un grupo electrógeno que aparece reflejada en el proyecto adjuntado a la solicitud formulada".

La sentencia concluye estableciendo: "En conclusión, pese a que se trata de una motivación ciertamente no muy sólida, lo cierto es que ni se puede estimar plenamente acreditado que la ubicación haya de ser la que interesa la parte y que no sea posible fuera del DPMT, ni sólo el informe desfavorable por sí solo determinaría justificación suficiente, aunque es cierta la existencia del informe desfavorable realizado por el Ayuntamiento de Arona y que en el mismo se indica que existe una oferta de este tipo de actividades en el tramo de costa que no justifica la autorización solicitada. Ha de tenerse en cuenta que el art. $61.2, b)$ no sólo habla sólo de la configuración del tramo de costa, sino también de que en dicho tramo resulte necesario su emplazamiento, esa necesidad no concurre en el presente caso".

\section{4.- AUTORIZACION AMBIENTAL INTEGRADA DE CENTRAL DIESEL.}

La sentencia de 19 de marzo de 2018 analiza el recurso presentado contra la autorización ambiental integrada de la instalación denominada "Central Diesel Punta Grande", situada en el término municipal de Arrecife, Isla de Lanzarote.

Para entender el presente recurso, han de tenerse en cuenta los siguientes antecedentes:

(1) Por resolución no 164/2008, del Viceconsejero de Medio Ambiente, se otorgó Autorización Ambiental Integrada de la instalación "Central Diesel Punta Grande", situada en el término municipal de Arrecife, incluida su ampliación mediante dos grupos diesel de 18,5 MW de potencia eléctrica unitaria, numerados con grupos 9 y 10

Y contra dicha resolución se interpuso recurso contenciosoadministrativo por la Asociación Las Caletas para la Defensa del Medio Ambiente (en adelante ACAPAM) que se siguió en esta Sala con el $n^{\circ}$ 
192/09, y en el que se dictó sentencia, con fecha de 2 de mayo de 2011, que declaró la nulidad de la resolución recurrida, siendo confirmada dicha Sentencia en casación por Sentencia del Tribunal Supremo de 5 de mayo de 2.014 . .

(2) Estando en tramitación el recurso de casación, por resolución de la Viceconsejería de Medio Ambiente no 475/12, de 7 de noviembre de 2.012, se otorgó a la entidad Unión Eléctrica de Canarias S.A.U., nueva Autorización Ambiental Integrada para la instalación, incluida su ampliación mediante los grupos diésel 11,12 y 13.

Y contra esta resolución también interpuso ACAPAM recurso contencioso-administrativo ante la Sala seguido con el no 60/13, que finalizó con el desestimiento y archivo por Decreto de 9 de abril de 2.014 .

(3) En cumplimiento de lo previsto en la Disposición Transitoria Primera de la Ley 5/2013, de 11 de junio, se incoó procedimiento de actualización de la Autorización Ambiental Integrada, al que puso fin la resolución de la Viceconsejería de Medio Ambiente de 8 de enero de 2.014, que actualizó dicha Autorización Ambiental Integrada de la instalación denominada "Central Diesel Punta Grande", para su adecuación a la Directiva 2010/75/CE, del Parlamento Europeo y del Consejo, de 24 de noviembre, sobre las emisiones industriales, y junto con ello, se dejó sin efecto la otorgada por resolución de 7 de noviembre de 2.012. .

\section{A partir de lo expuesto, los recurrentes basan s recurso en los siguientes puntos:}

a) Por cuanto con la Sentencia de esta Sala, confirmada en casación, que declaró la nulidad de la resolución n 164/2008, del Viceconsejero de Medio Ambiente, desapareció la cobertura jurídica que amparaba la actividad de la Central por incumplir las determinaciones urbanísticas que impedían el otorgamiento de la autorización, de forma que los mismos incumplimientos se trasladaron a la nueva autorización, otorgada por resolución $n^{\circ} 475 / 12$ (AAl de 2.012) y a la actualización aquí recurrida, y ello por cuanto continua vigente la misma ordenación territorial y urbanística (Plan Insular y Plan General) que llevó a la Sala y al Tribunal Supremo a declarar su incompatibilidad con la ordenación territorial y urbanística.

Explica la parte al respecto, que tanto la autorización concedida por resolución $n^{\circ} 475 / 12$ como la actualización no tienen otra finalidad que intentar volver a dar cobertura jurídica a la actividad de la central que ya se había perdido, y, en definitiva, a eludir los efectos de una sentencia firme, por lo que la resolución ahora impugnada incurre en reserva de dispensación y en desviación de poder, concluyendo que no ha existido, en el caso, una legalidad sobrevenida que imposibilitase la ejecución de la Sentencia sino "hecha venir a propósito para impedir la ejecución de la sentencia originaria, y ello no puede ser considerado sino un uso desviado de las potestades que a la Administración corresponden, es decir, que por la Administración demandada se incurre en los vicios de desviación de poder y reserva de dispensación que convierten en ilegal la nueva AA1 que debe ser anulada",

b) Por ser la instalación contraria a las determinaciones sobre 
Producción de Energía Eléctrica del Plan Insular de Ordenación de Lanzarote, que prevé la necesidad de una nueva Central a medio plazo, y que establece la obligación, como Directriz Vinculante, que la ampliación de la Central Punta Grande venga precedida de un Plan Especial que aún no ha sido aprobado (art 3.4.4.2 de la normativa del Plan Insular bajo la rúbrica "Producción de Energía Electrica" y Directriz Vinculante del apdo C 2)

c) Por ser también contraria a la normativa del Plan General de Ordenación de Arrecife que respecto a los Sistemas Generales, entre los que se debe incluir la Central por venir así contemplado en el Plan Insular, solo permite obras de mantenimiento y mejora que no supongan cambios de uso formales, ni de volumen.

d) Por ausencia de informe urbanístico en relación con la compatibilidad de la actualización con el planeamiento que debieron emitir tanto el Ayuntamiento de Arrecife como el Cabildo de Lanzarote, advirtiendo que, aunque existe un informe de la Oficina del Plan Insular, no aborda las cuestiones referidas a la compatibilidad con el planeamiento insular de la Central.

e) Por incumplir el artículo 4 del Reglamento de Actividades Molestas, Insalubres, Nocivas y Peligrosas, aplicable en cumplimiento de la Disposición Transitoria Cuarta de la Ley 1/1998, de 8 de enero, de Régimen Jurídico de los Espectáculos Públicos y Actividades Clasificadas. que establece que solo podrán emplazarse a una distancia de 2.000 metros al núcleo de población mas próximo,

f) Por cuanto no se han cumplido por la entidad explotadora las medidas impuestas en la Autorización Ambiental que se actualiza como se hace constar en informe técnico municipal de 21 de febrero de 2.013,

g) Por ser contraria a derecho la instalación como consecuencia de la saturación de SO2 y partículas contaminantes que se produce en la zona de Punta Grande al existir otros ocho grupos generadores de energía eléctrica y dos turbinas de gas., con vulneración de los límites de emisión establecidos en el Real Decreto 833/1975, de 6 de febrero.

h) Por no garantizar el cumplimiento de los niveles máximos de contaminación acústica de las Ordenanzas municipales y del Real Decreto 1367/2007.

i) Por incumplimiento de los condicionantes para la autorización de puesta en funcionamiento.

El Tribunal comienza a analizar cada una de las alegaciones realizadas por los recurrentes. Así, en cuanto a la cuestión de fondo, la Sala parte, en primer lugar, de la existencia de una resolución firme en vía administrativa y consentida, que no es otra que la resolución $n^{\circ} 475 / 12$, de otorgamiento de la Autorización Ambiental Integrada (AAI de 2012). Dicha firmeza deriva del desistimiento aceptado por la Sala y acordado por Decreto, del recurso contencioso-administrativo interpuesto contra dicha resolución. También debe advertirse, como segunda aproximación, que la resolución recurrida, de actualización de la Autorización Ambiental Integrada otorgada por la resolución 
$n^{0} 475 / 12$, trae causa en lo dispuesto en la Disposición Transitoria Primera de la Ley 5/2013, de 11 de junio, por la que se modifican la Ley 16/2002, de 1 de julio, de prevención y control integrados de la contaminación y la Ley 22/2011, de 28 de julio, de residuos y suelos contaminados. En consecuencia, entiende el Tribunal que pese a que gran parte del esfuerzo argumental de la Asociación demandante se dirige a poner de relieve la ausencia de compatibilidad de la Autorización Ambiental Integrada de la Central Diesel Punta Grande y de sus ampliaciones con la normativa territorial y urbanística, esto es, la incompatibilidad con la normativa del Plan Insular de Lanzarote sobre producción de energía eléctrica y con la normativa del Plan General sobre usos permitidos, la Resolución del Tribunal entiende que, por el contrario, "tal examen de legalidad debe hacerse en relación con la actualización de una Autorización Ambiental ya otorgada, y cuyo otorgamiento es un acto firme en vía administrativa al no haber sido recurrido en sede judicial ( situación que, en el caso, deriva del desistimiento del recurso aceptado por Decreto judicial firme)". Y ello, porque, "Entender lo contrario, es decir, examinar la legalidad de lo que es el contenido - urbanístico y no urbanístico- de la Autorización Ambiental Integrada otorgada por la Resolución del Viceconsejero del Medio Ambiente $n^{\circ} 475 / 12$, supondría revivir la posibilidad de impugnación de un acto firme en vía administrativa que tiene su particular cauce de impugnación en la legislación básica del procedimiento administrativo, que no es otro que los recursos referidos a la revisión de los actos firmes en vía administrativa".

A partir de dicha afirmación la sentencia centra su análisis en dos cuestiones: la vulneración de las disposiciones urbanísticas, y la desviación de poder. En relación con las primeras, la sentencia señala que lo que se alega es la ausencia de informes urbanísticos como irregularidad invalidante, a lo que responde que "frente a ello cabe decir que estamos ante un procedimiento especial diseñado por la Ley 5/2013 que, en palabras de su Exposición de Motivos, y a los efectos de garantizar la transposición adecuada de la Directiva 2010/75/UE, de 24 de noviembre, sobre Emisiones Industriales, establece, como régimen transitorio, un procedimiento de actualización de las autorizaciones ya otorgadas, en virtud del cual, el órgano ambiental competente de oficio comprobará, mediante un procedimiento simplificado, la 
adecuación de la autorización a las prescripciones de la nueva Directiva, con el 7 de enero de 2.014 como fecha límite para la actualización de las autorizaciones".

Se trata, continúa el Tribunal, "de un procedimiento simplificado que no exige informes urbanísticos, que si son necesarios en relación con la Autorización Ambiental otorgada que se actualiza conforme al artículo 15 de la Ley 16/2002, pero no para la actualización. Si concluyésemos que no existen dichos informes, son insuficientes, o no se acomodan a la normativa urbanística estaríamos anulando una actualización que no precisa dichos informes, y, a la vez estaríamos examinando la legalidad, desde una perspectiva urbanística, de un procedimiento distinto (el procedimiento para el otorgamiento de una Autorización Ambiental y no el procedimiento para su actualización), sin perjuicio de lo cual se ha aportado un certificado urbanístico del Ayuntamiento de Arrecife de fecha 2 de febrero de 2.015, que incluye un informe de la Arquitecta municipal de 30 de enero de 2.015, que concluye que la actividad de la Central Eléctrica de Punta Grande es compatible con el vigente Plan General, y lo mismo explicó el técnico de la Oficina del Plan Insular en relación con el PIO.

A continuación, se da respuesta al resto de los motivos, con un componente evidentemente técnico, remitiendo a las respuestas que en la Orden Departamental que resolvió el recurso de alzada sin que la parte recurrente haya traído a debate motivo alguno de nulidad de dichas conclusiones.

Lo que sí interesa en este momento es traer a colación la respuesta que el Tribunal da respecto de la naturaleza y efectos de la Autorización Ambiental Integrada. A este respecto, recuerda, en primer término, "que la autorización es un acto que supone un control preventivo o inicial, sin perjuicio de lo cual va acompañada de un conjunto de medidas que permiten el seguimiento del cumplimiento de los condicionantes impuestos".

Al respecto, recuerda que "la propia resolución de actualización advierte que "(..) queda condicionada a los límites y condicionantes técnicos recogidos en los Anexos a la presente resolución que forman parte inseparable de la misma" (apdo. tercero de su parte dispositiva) y que en caso de incumplimiento de las 
condiciones de la autorización ambiental, se añade en el apdo. quinto que "El órgano ambiental así como la administración competente en la materia objeto de incumplimiento, podrá ordenar al titular que ajuste su actividad a las normas y condiciones establecidas, fijando un plazo adecuado para ello, y, asimismo, exigir que el titular adopte las medidas complementarias necesarias para evitar o minimizar las molestias o los riesgos o daños que dicho incumplimiento puede ocasionar en el medio ambiente y la salud de las personas"

A lo que se añade que como medida de intervención se establece que "En caso de que el incumplimiento de las normas ambientales o de las condiciones establecidas en la autorización suponga un peligro inminente para la salud humana o amenace con causar un efecto nocivo inmediato significativo en el medio ambiente y en tanto no pueda volver a asegurarse el cumplimiento de las exigencias de la autorización, se podrá suspender la explotación de las instalaciones o de la parte correspondiente, de acuerdo con lo establecido en la Ley 16/2002, de 1 de julio". Y, por otra parte, en el apdo noveno se prevé, como medida más restrictiva, la posibilidad de revocación de la autorización por incumplimiento de las condiciones establecidas o de los requisitos legales para el ejercicio de la actividad.

Ante todo ello, la sentencia añade que "centrado el examen en lo que es la concurrencia de los requisitos para la autorización ambiental, ello es plenamente compatible con la posibilidad de corrección, suspensión de actividad o revocación en caso de incumplimiento de los condicionantes y garantías de la autorización, si bien se trata de una cuestión ajena al presente proceso que examina si se cumplían los requisitos de la actualización respecto a los cuales la parte demandante no ha justificado su incumplimiento".

Finalmente resulte interesante la reflexión final que añade respecto de la naturaleza de la Autorización Ambiental Integrada: "En este sentido, y conforme a la interpretación auténtica del concepto de Autorización ambiental Integrada de la Ley 5/2013, estamos ante una resolución escrita del órgano competente de la Comunidad Autónoma en la que se ubique la instalación, por la que se permite, a los efectos de la protección del medio ambiente y de la salud de las personas, explotar la totalidad o parte de una instalación, bajo determinadas condiciones destinadas a garantizar que la misma cumple el 
objeto y las disposiciones de la ley. Otra cosa será el incumplimiento de esas condiciones lo cual permite, dentro de lo que es el control continuado del ejercicio de la actividad, un conjunto de medidas a las que hace especial referencia la propia actualización que pueden llegar, como medida más intensa de las de las posibles, a la revocación". 